\title{
Photonic Sensor System for Screening Serum Biomarker Proteins in Ovarian Cancer
}

\author{
Debra Wawro ${ }^{1}$, Shelby Zimmerman 1 , \\ Robert Magnusson ${ }^{1,2}$ and Peter Koulen ${ }^{3}$ \\ ${ }^{1}$ Resonant Sensors Incorporated (RSI), Arlington, TX \\ 2University of Texas at Arlington, Department of Electrical Engineering, Arlington, TX \\ ${ }^{3}$ University of Missouri - Kansas City, School of Medicine, Departments of \\ Ophthalmology and Basic Medical Science, Vision Research Center, Kansas City, MO
}

USA

\section{Introduction}

Ovarian cancer is among the most deadly types of cancers among women, with about 21,990 new cases diagnosed every year in the United States (American Cancer Society, 2011). About 15,460 of these women will die from ovarian cancer. If diagnosed while the cancer is still localized, survival rates of at least 5 years are likely. Unfortunately, less than $20 \%$ of cases are found at an early stage due to the absence of reproducible and definitive diagnostic tools. Because ovarian cancers occur deep in the pelvis, there are often few symptoms until the cancer is at an advanced stage. Furthermore, many of the symptoms of ovarian cancer (such as back pain, fatigue, and abdominal bloat) are common and difficult to distinguish from those not caused by cancer. Because of this lack of symptom specificity, most ovarian cancers are substantially advanced at the time of diagnosis. Staging of the cancer is critically important in order to determine the most effective treatment modality. Currently there are no routine clinical diagnostic assays using urinalysis or seranalysis for early screening or staging of ovarian cancer. However, there are several research studies (Bignotti et al., 2007; and Liotta et al., 2005) that identify potential biomarker indicators that can be used for this purpose.

When a woman is suspected of having ovarian cancer, medical diagnostics typically include an ultrasound of the abdomen and pelvis as well as a blood test that includes measurement of the CA-125 protein levels (American Cancer Society, 2011). CA 125 is a protein biomarker found in greater concentration in tumor cells than in other cells of the body. However, since CA-125 levels can be elevated due to other benign causes, it is primarily used to monitor women with a known cancer of the ovary to determine treatment efficacy. Measurement of CA-125 levels is not accepted as a sufficient test for an early screening indicator in ovarian cancer. Thus, improved methods are needed to provide a specific and early screen for this deadly disease.

Based on "Optical nanotechnology enables rapid label-free diagnostics for cancer biomarker screening," by D. Wawro, S. Zimmerman, R. Magnusson and P. Koulen which appeared in Proceedings of SPIE 8090, 80900 (2011). 
In this work, we describe a high-accuracy, label-free biosensor system that can provide effective detection of an array of biomarker proteins in serum to accurately diagnose and stage ovarian cancer. While there are currently no established clinical diagnostic assays using urinalysis or seranalysis, experimentally and clinically identified targets (Bignotti et al., 2007; and Liotta et al., 2005) can be categorized into two groups: group 1 consists of biomarker proteins that are up-regulated twofold or higher in metastatic over primary ovarian serous papillary carcinoma (such as Fibronectin), and group 2 consists of biomarker proteins that are up-regulated twofold or higher in primary over metastatic ovarian serous papillary carcinoma (such as Apolipoprotein A-1). This differentiation yields accurate diagnosis of the disease and staging information that can be used to monitor presymptomatic aspects of the disease, disease progression, and the efficacy of therapies.

Conventional blood diagnostic testing methods such as immunoassay approaches require time-intensive processing and washing steps, and they are not easily integrated in a clinical setting. To address these needs, we utilize a real-time photonic biosensor technology that provides rapid results with minimal processing steps and the capability to test for an array of biomarkers in a single sample.

\section{Label-free diagnostic approach}

The diagnostic screening system that is central to this work applies an optical approach based on the guided-mode resonance (GMR) effect that occurs in subwavelength dielectric waveguide gratings. As shown in Fig. 1, when these diffractive elements are illuminated with a broadband light source, a specific wavelength of light is reflected (or transmitted) at a specific angle. The binding interaction between an immobilized receptor and its analyte can be monitored in real time without the use of reporter labels (such as fluorescent or radioactive tags) by following the corresponding resonance wavelength shift with an optical

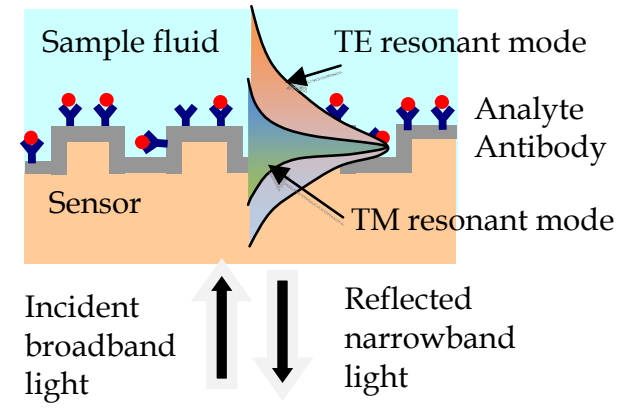

(a)

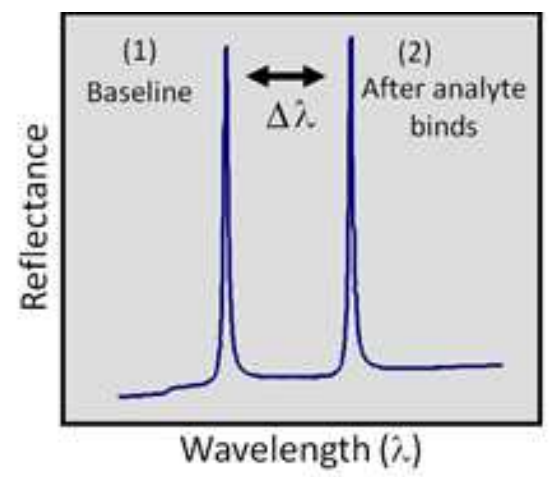

(b)

Fig. 1. (a) Schematic of a label-free GMR sensor system (single channel illustrated) operating in reflection mode. The collimated beam from a broadband source is incident on the sensor at normal incidence. The reflected spectral response is monitored in real time with an optical spectrum analyzer. (b) As binding events occur at the sensor surface, resonance peak changes (only one polarization depicted in plot) can be tracked as a function of the wavelength. 
spectrum analyzer. Test time is limited solely by the chemical binding dynamics between the receptor and its target. Specificity is imparted on the sensor surface by covalently attaching a selective layer (such as antibodies). It is multifunctional as only the sensitizing surface layer needs to be chemically altered to detect different targets. Repeatable fabrication processes are in place to produce the resonant grating sensor element in low-cost polymer and other dielectric materials.

Since the resonance layer is polarization-sensitive, separate resonance peaks occur for incident TE (electric vector normal to the plane of incidence) and TM (magnetic vector normal to the plane of incidence) polarization states. This dual-peak feature provides crossreferenced data useful for increasing detection accuracy. These distinct resonant modes interact differently with the surrounding media, enabling the polarization-based differentiation. This sensor technology is broadly applicable to medical diagnostics, drug discovery and development, industrial process control, and environmental monitoring.

\subsection{Guided-mode resonance technology overview}

The coupling of a freely propagating electromagnetic wave to a state of confinement at a periodic surface is presently the subject of considerable research activity. Periodic structures with subwavelength features provide effective means of achieving such coupling. The resulting strong localization of energy at a dielectric (or metallic) layer is of interest for numerous photonic applications including biosensors, light sources, nonlinear frequency converters, and particle traps. Magnusson et al. disclosed GMR filters that were tunable on variation in resonance structure parameters (Magnusson \& Wang, 1992). Wawro et al. presented GMR biosensor embodiments as well as system architectures (Wawro et al., 2000). Thus, spectral or angular variations induced via layer thickness change or on change in refractive index in surrounding media or in device layers can be used to sense these changes (Magnusson et al., 2011; Wawro et al., 2006; 2010). Additional aspects of GMR sensors in various applications have been discussed in the literature (Cunningham et al., 2002; Kikuta et al., 2001).

Thin-film structures containing waveguide layers and periodic elements, under the correct conditions, exhibit the GMR effect. Most commonly, GMR biosensors are designed to operate in reflection. In this configuration, an incident wave is phase-matched, by the periodic element, to a leaky waveguide mode. It is reradiated in the specular-reflection direction as it propagates along the waveguide and constructively interferes with the directly reflected wave. Conversely and equivalently, the phase of the reradiated leaky mode in the forward, directly transmitted wave direction is $\pi$ radians out of phase with the direct unguided transmitted wave, thereby extinguishing the transmitted light (Rosenblatt, 1997). This picture of the resonance effect pertains to a reflection, or bandstop, filter. Other operation configurations are possible, such as in transmission mode, or as a bandpass filter.

Figure 2 shows the measured and calculated spectral reflectance of a dielectric GMR device (Priambodo et al., 2003). It acts as a bandstop filter with the spectrum of interest reflected in a narrow band with relatively low sidebands. Although the theoretical calculation predicts $100 \%$ peak efficiency for a plane wave incidence, it is diminished in practice by various factors such as material and scattering losses, incident beam divergence, and the lateral device size; here, the experimental peak is $90 \%$. 
These resonant structures, tunable on change of refractive index and/or thickness, have clear applications for biosensors. The buildup of the attaching biolayer can be monitored in real time, without use of chemical tags, by following the corresponding resonance shift.

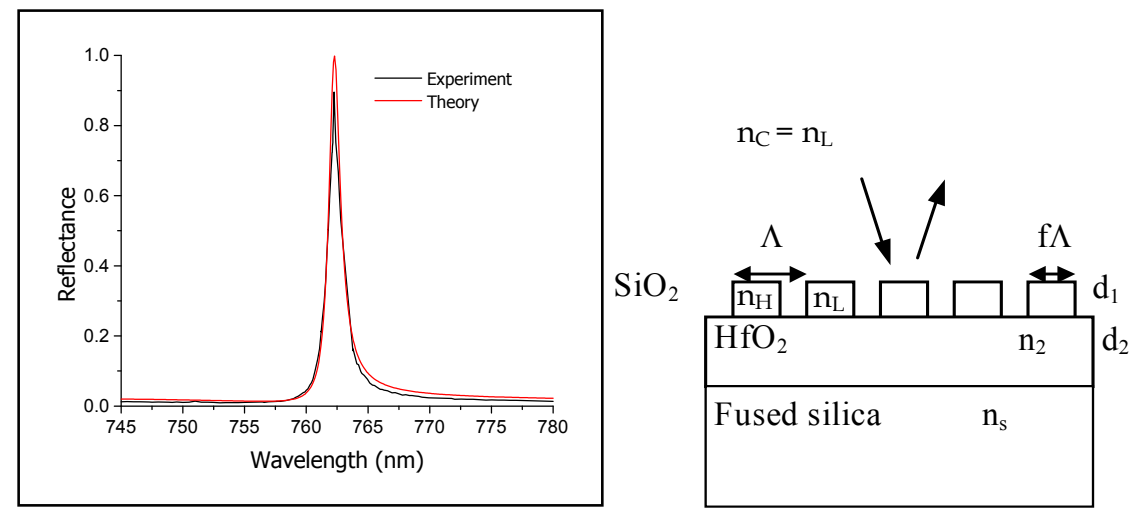

Fig. 2. Comparison between experiment and theory for a dielectric resonance element. The parameters used for the theoretical curve fit are close to the nominal values; they are $\mathrm{n}_{\mathrm{C}}=1.0$, $\mathrm{nH}=1.454$ (SiO2), n2=1.975 (HfO2), ns=1.454, d1=135 nm, f=0.58, d2=208 nm, $\Lambda=446 \mathrm{~nm}$, normal incidence. Rigorous coupled-wave analysis (RCWA) is used for the computations (Gaylord \& Moharam, 1985).

\subsection{Biosensor operation}

In addition to the reflection/transmission properties of propagating electromagnetic waves, the near-field properties of resonant periodic lattices, including localization and fieldstrength enhancement, are of interest in sensor applications. The near-field patterns associated with a typical filter, similar to that in Fig. 2 in structure, are shown in Figs. 3 and 4 with a normally incident TE-polarized wave. Numerical results are obtained with rigorous coupled-wave analysis (RCWA) (Gaylord \& Moharam, 1985) to provide quantitative information on relative field strengths and spatial extents associated with the near fields. As shown in Fig. 3 , the $S_{0}$ wave $\left(S_{0}\right.$ denotes the electric field of the zero order) propagates with reflected-wave amplitude close to unity, producing the standing-wave pattern shown by interference with the unit-amplitude input wave used in our model. Thus, at resonance, most of the energy is reflected back. The evanescent, first-order diffracted waves $S_{1}$ and $S_{-1}$ constitute the counter-propagating leaky modes. We see that the maximum field value is located in the $\mathrm{HfO}_{2}$ layer with the evanescent tails gradually penetrating into the substrate and cover. Figure 4 shows the standing wave pattern formed by the counter-propagating $\mathrm{S}_{-1}$ and $S_{+1}$ waves at a certain instant of time; the field scale is color coded as shown. Since the $\mathrm{S}_{ \pm 1}$ space harmonics correspond to localized waves, they can be very strong at resonance; here, the field enhancement is $\sim x 10$ as seen in Fig. 3 . Depending on the level of grating modulation $\left(\Delta \varepsilon=\mathrm{n}_{H^{2}}-\mathrm{n}_{\mathrm{L}}^{2}\right)$, the field amplitude can range from $\sim \mathrm{x} 10-\mathrm{x} 1000$ in the layer relative to the input wave amplitude that represents a large increase in local intensity I $\sim \mathrm{S}^{2}$. The maximum amplitude of $S_{1}$ is approximately inversely proportional to the modulation strength. In general, small modulation implies narrow linewidth $\Delta \lambda$ and a large resonator $Q$ factor $Q=\lambda / \Delta \lambda$. 


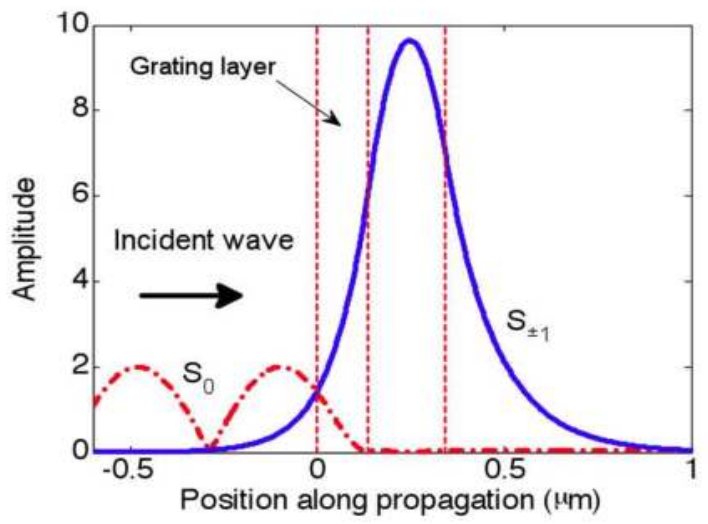

Fig. 3. Profile of the leaky mode at resonance for a typical GMR sensor device. The amplitude is normalized to the incident-wave amplitude.

The structure of the local fields associated with the resonant leaky modes is key to sensor applications. The leaky mode is a surface state that propagates along the surface, providing maximal interaction with any attached molecular or chemical layer. In the technology discussed herein, the sensing field (a resonant leaky mode) is maximized in the grating layer with an evanescent tail penetrating into the cover region (shown in Figs. 3 and 4).

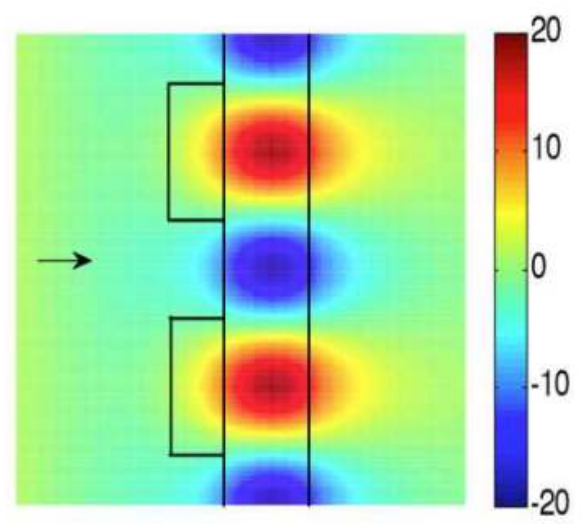

Fig. 4. Snapshot of the standing-wave pattern associated with the leaky mode in Fig. 3 . The size of the region is $2 \lambda \times 2 \Lambda$. Results are obtained with rigorous coupled wave analysis.

\subsection{Sensor element fabrication}

The GMR biosensor devices used in this work are based upon a single-layer waveguide grating design. We fabricated these with low-cost submicron molding methods in our labs, and they can be purchased from numerous commercial sources. We utilize polymers that are imprinted with submicron grating patterns and coated with a high-index dielectric material (such as $\mathrm{TiO}_{2}$ or $\mathrm{HfO}_{2}$ ) to realize resonant sensors. Figure 5 shows an example of a GMR sensor. 


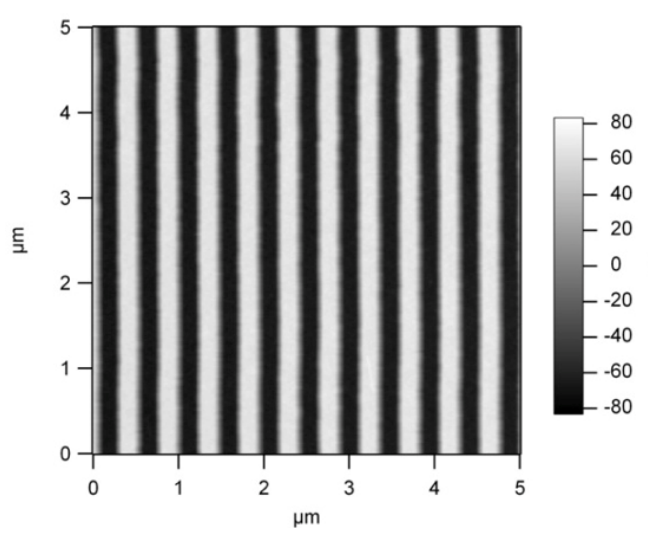

(a)

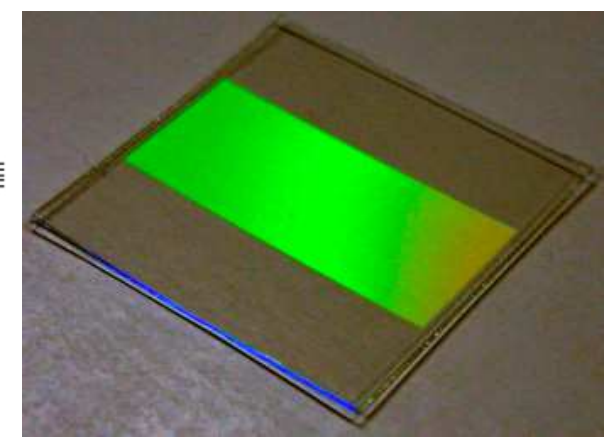

(b)

Fig. 5. Submicron resonant grating. (a) Atomic force microscope (AFM) picture of a 520-nm period grating contact printed in an optical polymer. (b) A picture of a submicron molded grating. The grating is coated with a thin high-index layer $\left(\mathrm{TiO}_{2}\right.$ or $\left.\mathrm{HfO}_{2}\right)$ to realize a GMR sensor element.

\subsection{Competing approaches}

Numerous optical sensors for bio- and chemical detection have been developed commercially and in research literature. Key label-free technologies include the surfaceplasmon resonance sensor (Homola, 2003; Raether, 1988), MEMS-based sensors, nanosensors (rods and particles), resonant mirror, Bragg grating sensors, waveguide sensors, waveguide interferometric sensors, ellipsometry, and grating coupled sensors (Cunningham, 1998; Cooper, 2006). Other methods include immunomagnetic separation, polymerase chain reaction, and standard immunoassay approaches that incorporate fluorescent, absorptive, radioactive, and luminescence labels. The GMR sensor approach has advantages and distinctions relative to these technologies, including features such as polarization diversity and low-power, portable system formats.

In our opinion, although dramatically different in concept and function, the surfaceplasmon resonance (SPR) sensor (Homola, 2003; Raether, 1988) comes closest in features and operation to the GMR sensor discussed here. The term surface plasmon (SP) refers to an electromagnetic field charge-density oscillation that can occur at the interface between a conductor and a dielectric (for example, gold/glass interface). An SP mode can be resonantly excited by parallel-polarized (TM, electric vector in the plane of the page) incident light but not with TE polarized light. Phase matching occurs by employing a metallized diffraction grating, or by using total internal reflection from a high-index material, such as in prism coupling or an evanescent field from a guided wave. When an SPR surface wave is excited, an absorption minimum occurs in a specific wavelength band. Since only a single polarization (TM) can physically be used for detection, refractive index and thickness attachments cannot simultaneously be resolved in one measurement. This is particularly important in chemical sensor applications where binding kinetics includes conformational and density changes at the sensor surface.

Standard label-based immunoassay tests involve extensive and complicated incubation and washing steps. In this approach, results are not obtained until 4-24 hours after starting the 
test. By using GMR sensor technology, real-time results can be obtained with no required washing steps. Results are limited only by the binding dynamics of the ligand-receptor interactions (typically less than 30 minutes). This greatly simplifies medical diagnostic testing approaches, and it will enable doctor offices and hospitals to perform routine screening on a much larger scale with dramatically less labor.

\section{Experiments}

Numerous characterization experiments have been performed for a variety of biological and chemical materials utilizing GMR sensors and the Vides bioassay spectroscopic reader system developed by Resonant Sensors Incorporated (shown in Fig. 6). In this work, we evaluate this label-free screening tool for the detection of biomarker proteins fibronectin and apolipoprotein A-1 (ApoA-1), which are relevant in ovarian cancer. The sensor plate (shown in Fig. 6(b)) is incorporated in the bottom of a bottomless microarray plate. Each well is sensitized to detect a target analyte by immobilizing a selective layer (such as highly specific antibodies). The spectroscopic sensor system approach (as shown in Fig. 1) tracks the GMR resonance peak wavelength changes as a function of time during a biochemical interaction. The relative peak shift is correlated to a concentration for a particular analyte in a serum or cell culture sample. We use an in vitro cell model for ovarian cancer to provide the relevant expressed biomarker proteins under test. Additionally, we investigate the impact of nonspecific binding and cross reactivity in complex samples such as human serum and cell media.
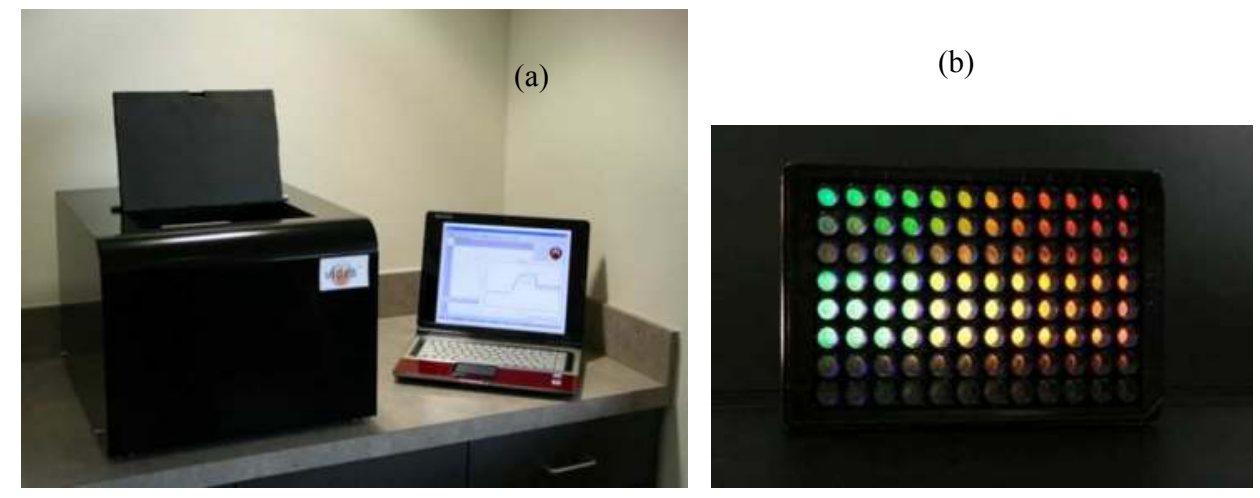

Fig. 6. (a) A benchtop spectroscopic detection system utilizing GMR biosensor technology developed by Resonant Sensors Incorporated (RSI). In this arrangement, the spectral reflectance is monitored with an optical spectrum analyzer, and the peak wavelength is tracked as a function of time during a biochemical event. (b) This bioassay reader utilizes 96well (shown here) or 384-well (not shown) sensor array plates.

\subsection{In vitro cell model}

Human cell lines are used for the detection of relevant biomarker proteins and feasibility of sensor operation in complex samples. In order to combine the highest possible clinical relevance for the most financially viable research plan, the in vitro models for ovarian cancer 
are chosen based on human cell lines that had been derived directly from patients with ovarian cancer and are not from other types of cancer with ovarian side effects/metastases. Additionally, the in vitro models are established (used by ovarian cancer researchers in peerreviewed publications) and reproducible (available through ATCC).

Two different cell lines are used to provide samples for the detection of ovarian cancer biomarker proteins as shown in Table 1. The cell culture supernatant, which contains the expressed biomarker proteins, is measured to determine the concentrations of fibronectin and apolipoprotein A-1 (detailed in next section). We culture both cell lines as follows:

\subsubsection{Cell culture growth}

Cells are thawed and transferred to a $15 \mathrm{ml}$ conical tube. Cells are spun at $200 \times \mathrm{g}$ for 1 minute. The supernatant is removed and replaced with $1 \mathrm{ml}$ of Complete Medium (MCDB 105 and Medium 199, with fetal bovine serum). A cell count is done on the Nexcelom T4 Cellometer (Nexcelom Bioscience LLC, Lawrence, MA). Cells are then seeded in two $75 \mathrm{~cm}^{2}$ flasks per vial of cells.

\begin{tabular}{|l|l|l|l|l|}
\hline Name & description & source & ATCC \# & Ref. \\
\hline Caov-3 & $\begin{array}{l}\text { Epithelial ovarian papillary } \\
\text { adenocarcinoma }\end{array}$ & human & HTB-75 & (Karlan \& Lagasse, 1994) \\
\hline TOV-21G & $\begin{array}{l}\text { Epithelial ovarian poorly } \\
\text { differentiated primary } \\
\text { malignant adenocarcinoma; } \\
\text { Stage IIIC }\end{array}$ & human & CRL-11730 & (Provencher et al., 1993) \\
\hline
\end{tabular}

Table 1. Ovarian cancer cell lines used in this work.

\subsubsection{Sub-culturing or passage}

The media is removed and collected for supernatant. The media is replaced with $0.25 \%$ trypsin/EDTA, and the flask is placed in an incubator for approximately 3-5 minutes. Once cells are detached, the suspension is removed and placed in a $15 \mathrm{ml}$ Falcon tube. The cell suspension is spun at $200 \times \mathrm{g}$ for 1 minute. The trypsin is removed, and the cell pellet is resuspended in Complete Medium (amount varies depending on confluence). The suspension is seeded into a fresh flask.

\subsubsection{Supernatant collection}

To collect supernatant, the media is removed from the culture flask, placed in $50 \mathrm{ml}$ Falcon tube, and spun at $300 \times \mathrm{g}$ for 1 minute. The supernatant is removed and placed in a fresh 50 $\mathrm{ml}$ falcon tube. The tubes are then frozen at $-80^{\circ} \mathrm{C}$.

\subsection{Protein biomarker screening}

Detection of the proteins fibronectin and ApoA-1 are performed in a variety of sample backgrounds, including a reagent diluent (containing bovine serum albumin, BSA), human serum, cell media, and cell culture supernatant. Figure 7 illustrates the spectral resonance peak shifts due to the binding of the ovarian cancer biomarker fibronectin in various concentrations. Fibronectin is a high-molecular weight $(\sim 440 \mathrm{kDa})$ extracellular matrix 
glycoprotein that is known to be produced by some ovarian cancer cell lines. To provide selectivity to fibronectin, anti-fibronectin monoclonal antibodies are immobilized on the sensor surface using commercial silane surface chemistries and cross-linking agents. Known standard concentrations of the target analyte fibronectin are diluted in a reagent diluent solution in phosphate buffered saline (PBS, pH 7.4). This reagent provides a BSA blocking agent to minimize nonspecific binding during the reaction. Both TE and TM polarization resonances are tracked for each concentration. Neat reagent diluent is used as a reference blank and subtracted from the data in Fig. 7. Binding is monitored for 1 hour at $37^{\circ} \mathrm{C}$. At the end of the binding, any loose or unbound fibronectin is rinsed away in PBS, and a postbinding measurement is taken. Final data is shown using the relative peak shifts recorded pre- and post-binding in PBS. Both TE and TM resonances trend similarly, with the TM peak having slightly better detection sensitivity. The limit of detection for this assay is $\sim 20$ $\mathrm{ng} / \mathrm{ml}$.

\section{Fibronectin Detection in Reagent Diluent}

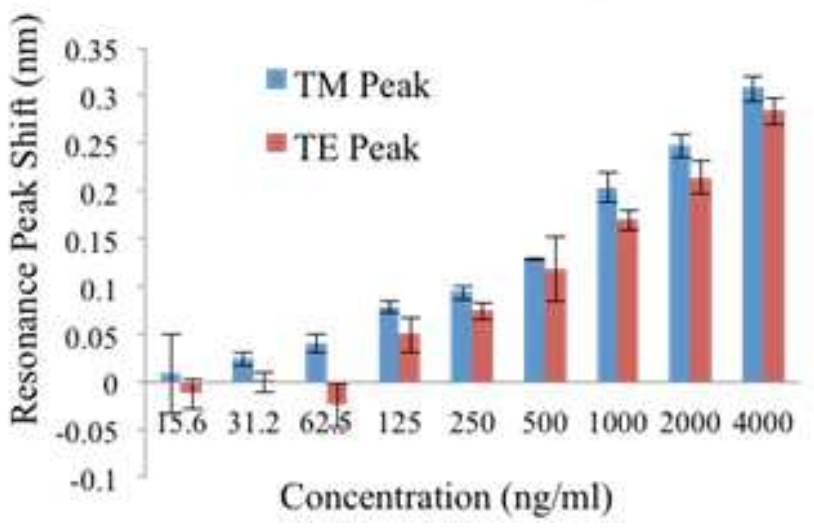

Fig. 7. Resonance peak shift as a function of concentration for fibronectin binding to its matched antibody on the sensor surface. Both TE and TM polarization resonances are tracked. Results are repeated in quadruplicate and averaged.

Figure 8 illustrates fibronectin detected in Caov-3 cell culture media and supernatant. The TM resonance peak shift for the test sample (unknown) is compared to the standard concentration (known) to obtain a measured concentration of $439.1 \mathrm{ng} / \mathrm{ml}$ for Caov-3 media and $996.7 \mathrm{ng} / \mathrm{ml}$ for Caov-3 supernatant. This indicates that the cell line is expressing fibronectin under culture conditions. Additional concentration measurements were performed for detection of fibronectin in TOV-21G media and cell culture supernatant. Summarized results comparing measured concentrations of both Caov-3 and TOV-21G are shown in Fig. 9. For TOV-21G (a stage IIIC ovarian cancer cell line), fibronectin levels are reduced during cell culture. 


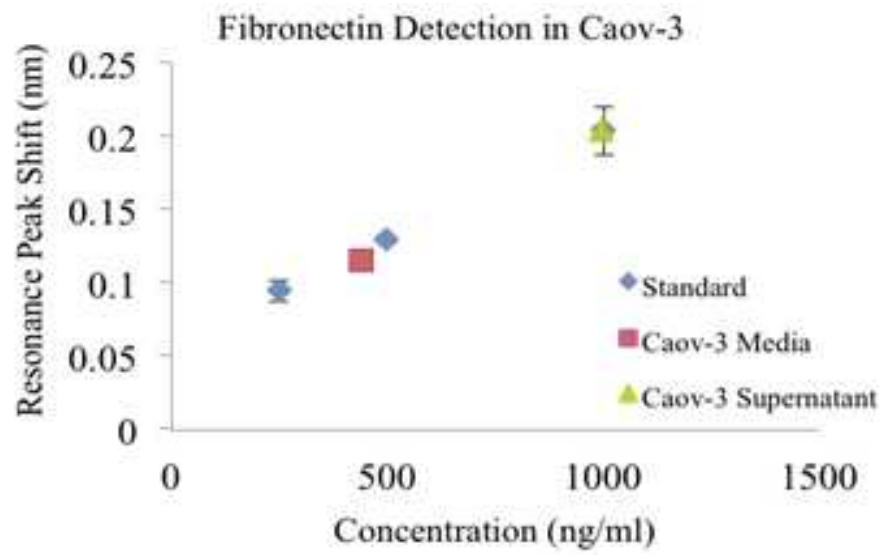

Fig. 8. Resonance peak shift as a function of concentration for detection of fibronectin.

Standards are generated in a reagent diluent background. Caov-3 supernatant (green) and media (red) sample resonance shifts are compared to the known concentration resonance shifts (standard curve in blue) to obtain Fibronectin concentrations. All measurements are repeated in quadruplicate and averaged. Some standard deviations are too small to display on chart.

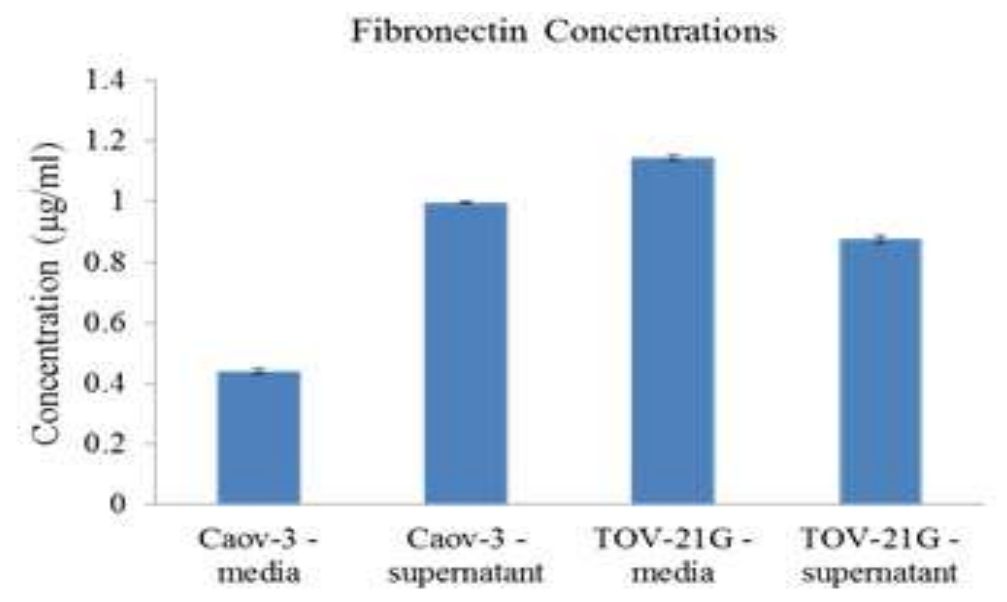

Fig. 9. Comparison of the measured fibronectin in cell culture media and expressed supernatant for two different ovarian cancer cell lines.

Detection of the expressed biomarker protein ApoA-1 in the ovarian cancer cell culture supernatant was also quantified for cell lines Caov-3 and TOV-21G. ApoA-1 is a protein component of high-density lipoprotein in plasma, and it has an approximate molecular weight of $28 \mathrm{kDa}$. In this experiment, anti-ApoA-1 antibodies are immobilized on the sensor surface to provide targeted selectivity for detection. Figure 10 illustrates measured TM-resonance shifts for standard known concentrations of ApoA-1 in reagent diluent (shown in blue). We also measure unknown amounts of ApoA-1 in fresh (unused) cell culture media and in ovarian cancer cell supernatant. The known standards are used to generate a linear calibration curve 
for the range from $32 \mathrm{ng} / \mathrm{ml}$ to $125 \mathrm{ng} / \mathrm{ml}$ (with an $\mathrm{R}_{2}$ value of 0.989). Based on this linear fit, the fresh cell culture media is found to contain $\sim 59 \mathrm{ng} / \mathrm{ml}$ ApoA- 1 while the cell culture supernatant contains $\sim 89 \mathrm{ng} / \mathrm{ml}$. Binding is monitored for 1 hour at $37^{\circ} \mathrm{C}$.

\section{Apolipoprotein A1 Detection in TOV-21G}

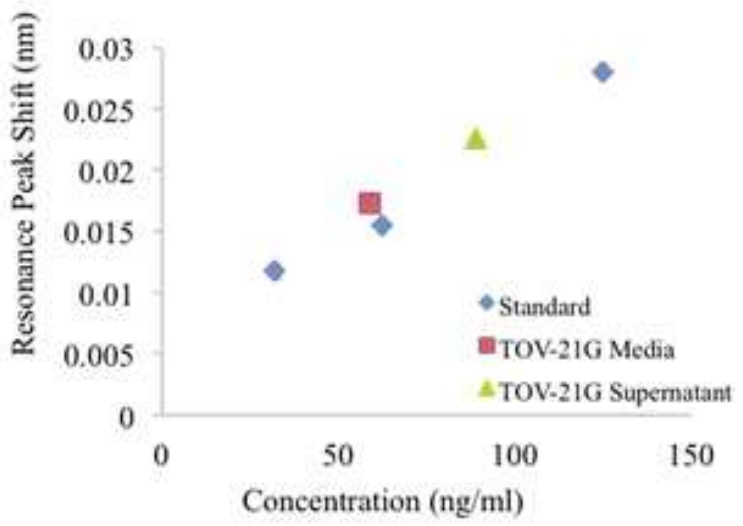

Fig. 10. Resonance peak shifts measured for detection of apolipoprotein A-1. Known standards are measured (shown in blue) to obtain a calibration curve that is used to quantify the unknown samples (shown in red and green). Samples are run in quadruplicate and averaged, with major outliers removed. Standard deviation is negligible (shown on plot).

Figure 11 illustrates detection of the biomarker ApoA-1 in culture media and supernatant for the Caov-3 and TOV-21G cell lines. The TM resonance peak shift for the test sample (unknown) is compared to the standard concentration (shown in Fig. 10) to obtain a measured concentration for each sample. Summarized results comparing measured concentrations of both cell media and culture supernatant are shown in Fig. 11. For the TOV-21G cell line, ApoA-1 is increased (or expressed) in the measured supernatant. In the Caov-3, the measured amount in the supernatant is reduced during culture. Tests are run in quadruplicate and averaged.

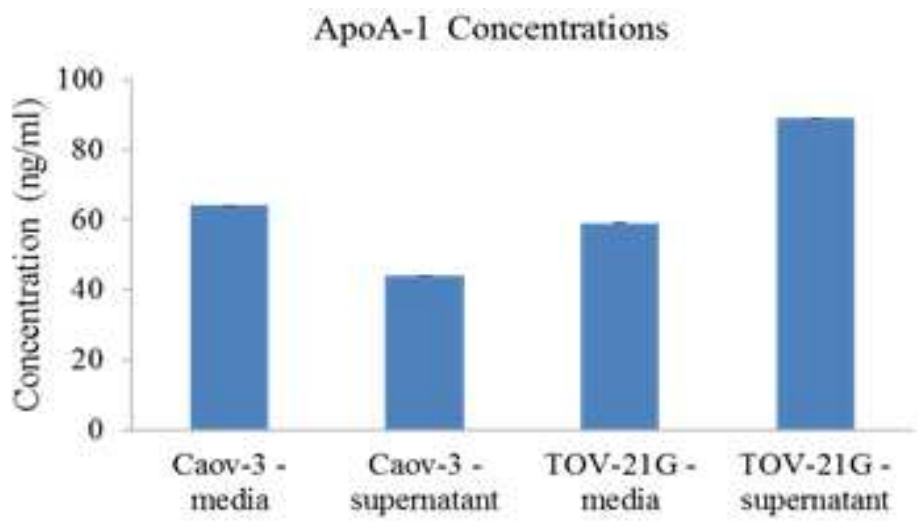

Fig. 11. Comparison of the measured ApoA-1 in cell culture media and expressed supernatant for two different ovarian cancer cell lines. Standard deviation is negligible (shown on plot). 


\subsection{Nonspecific binding}

To investigate the amount of nonspecific binding that might occur during the media/supernatant and serum experiments, we prepare a negative reference well using a blocked silanized well (no antibodies attached); it is compared to wells containing specific antibodies for ApoA-1 and fibronectin. The capture antibodies for ApoA-1 and fibronectin are monoclonal mouse antibodies that are chemically attached to the sensor surface using a silane-based crosslinking agent. After antibody attachment, the unbound sites are blocked with a blocking buffer (BSA). In Figure 12, a cell culture media sample (having ApoA-1 naturally present) is incubated (1 hour) on sensor wells containing antibodies specific for ApoA-1 and wells that have no antibodies present. Figure 12 illustrates the minimal shift results from the negative reference well (no antibodies) as compared to the well containing the specific antibodies (large shift). We also investigate the use of human serum as a sample background in the detection of fibronectin. Figure 13 illustrates the resonance peak shift results from a serum sample (naturally containing fibronectin) after incubation ( 1 hour) on a negative reference well (no antibodies) compared to the specific antibody coated region. Both of these results are based on the difference of initial and final PBS baseline readings.

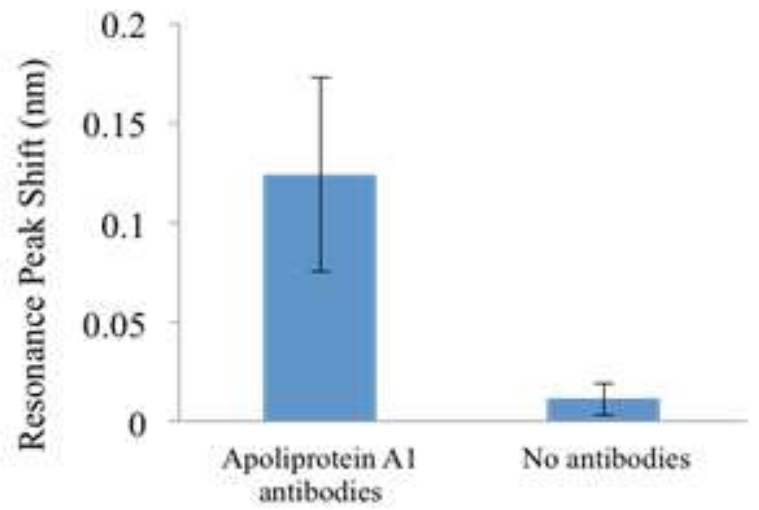

Fig. 12. Comparison of the TM resonant peak shift due to binding of the biomarker ApoA-1 (in a cell media background) to the ApoA-1 antibodies on the sensors surface versus nonspecific binding on the sensor elements not coated with antibodies.

Since the cell media and supernatant samples are made up of complex matrices, we used a spike and recovery method (Thermo, 2007) for each biomarker protein assay to determine whether the protein detection is affected by a difference between the diluent used to prepare the standard curve and the cell media sample matrix. In spike and recovery experiments, a known amount of protein standard is added to the sample matrix (corresponding growth media for each cell line) and compared to a standard curve measured in diluent. The two sets of total resonance peak shift measurements are compared. Table 2 shows results for spike and recovery experiments performed for fibronectin in both Caov-3 and TOV-21G cell culture media. Measurements are based on the difference of initial and final baseline readings with pure reagent diluent or pure media used as negative controls and subtracted from the data. In both cases, the detected amount was within $\sim 10 \%$ of the target. 


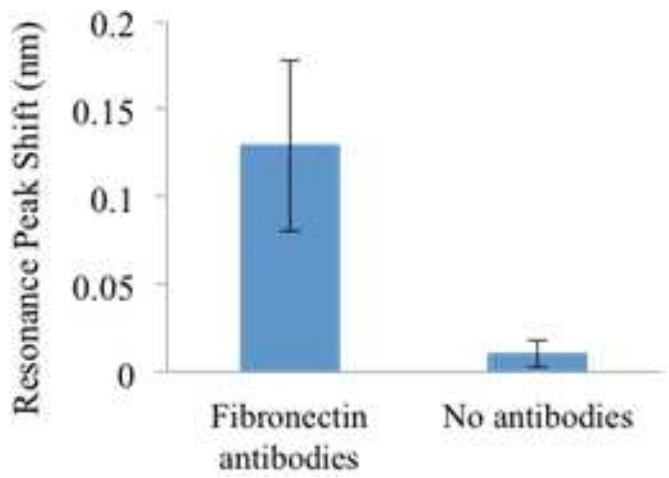

Fig. 13. Comparison of the TM resonant peak shift due to the binding of fibronectin (in human serum) to the fibronectin antibodies on the sensors surface versus the nonspecific binding on the sensor elements not coated with antibodies. Tests are run in quadruplicate and averaged.

\begin{tabular}{|l|l|l|l|l|}
\hline Medium & $\begin{array}{l}\text { Spike } \\
\text { (ng/ml) }\end{array}$ & $\begin{array}{l}\text { Standard Resonance } \\
\text { Peak Shift }(\mathbf{n m})\end{array}$ & $\begin{array}{l}\text { Spiked Resonance } \\
\text { Peak Shift }(\mathbf{n m})\end{array}$ & $\begin{array}{l}\text { Recovery } \\
\mathbf{0}\end{array}$ \\
\hline Caov-3 & 500 & 0.129 & 0.135 & 104.7 \\
\hline TOV-21G & 500 & 0.129 & 0.116 & 89.9 \\
\hline
\end{tabular}

Table 2. Fibronectin Spike and Recovery.

\section{Dual-peak analysis}

As shown in Fig. 1, there are separate resonance peaks for each polarization (TE and TM) that shift in response to a given measurement. By backfitting this dual-peak response into our rigorous electromagnetic coupled wave analysis codes (Gaylord \& Moharam, 1985), we can determine two unknowns: surface changes due to analyte binding and bulk refractiveindex changes that occur due to sample background variations. First, we calculate and map the predicted TE and TM resonance peak shifts over a relevant range of added biolayer thicknesses ( 0 to $50 \mathrm{~nm})$ and background index variations $(\mathrm{n}=1.33$ to $\mathrm{n}=1.5)$. A simple matrix is applied to match the corresponding detection layer and background index when the two resonance peak shifts are known. This data is fitted assuming a known biolayer refractive index, with unknown values to be determined for the biolayer thicknesses and background index. To illustrate the utility of this approach, we use the ionic polymer poly (allylamine hydrochloride) to study binding interactions that involve biolayer adhesion and associated thickness change at the sensor surface (Magnusson et al., 2011). Two resonance peaks are tracked as the ionic polymer attaches a monolayer of material as shown in Fig. 14. After the polymer saturates, the measurement is paused and the sensor is washed to remove any unbound polymer. A post-binding measurement is made in DI water. The results in Fig. 15 show that the binding of the polymer layer to the sensor surface contributes most to the measured response. The fitted background drift is partially attributed to thermal changes in the sample during the measurement and imperfect model assumptions (such as polymer layer index). Improvements to the backfit model will further distinguish these contributions. 


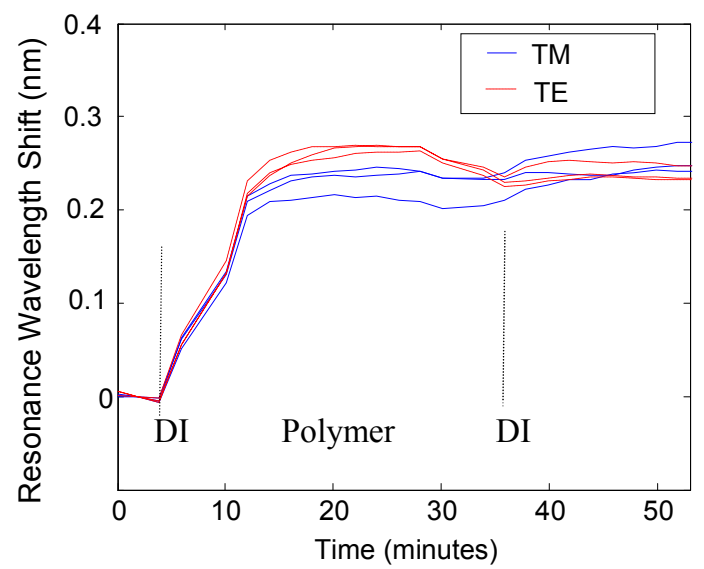

Fig. 14. Resonance peak shifts as a function of time for binding ionic polymer to the sensor surface. Both TE and TM resonances are monitored. This medium has a molecular weight of $56,000 \mathrm{kDa}$.

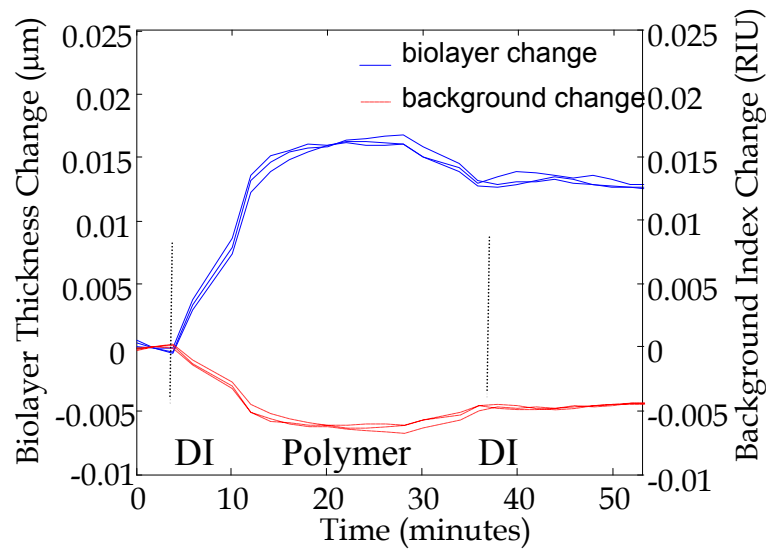

Fig. 15. Results of backfitting to a simple model, thereby differentiating contributions from biolayer adhesion and background changes.

\section{Conclusions}

A novel diagnostic system to detect biomarkers relevant for diagnosis of ovarian cancer has been developed. This label-free sensor system can accurately and rapidly detect an array of protein markers with minimal sample processing requirements. Sensor performance was characterized for the biomarker proteins fibronectin and apoliprotein A-1 with limits of detection measured to be $\sim 20 \mathrm{ng} / \mathrm{ml}$ in backgrounds of cell culture media and human serum. An in vitro cell culture model was used with established ovarian cancer cell lines to provide relevant samples for this work. Nonspecific binding effects were investigated for operation in serum backgrounds with minimal impact. Additionally, due to inherent 
polarization diversity, these sensors employ multiple resonance peaks that are used to increase detection accuracy by providing multiple data points for each test. Work is ongoing to integrate this system into a portable detection unit that can be used in a point-of-care setting. Future work will include clinical sample validation and an expanded array of relevant biomarkers that can be tested in a single sample. This will provide a highly accurate rapid screening tool for early detection of ovarian cancer.

\section{Acknowledgements}

This work was supported in part by the National Science Foundation SBIR grant \#0724407 (D.W), the National Cancer Institute SBIR grant \#R43CA135960 (D.W. and P.K.) and the State of Texas Emerging Technology Fund (D.W.). Additional support was provided by the UT System Texas Nanoelectronics Research Superiority Award (R.M.), the Texas Instruments Distinguished University Chair in Nanoelectronics endowment (R.M.), the Vision Research Foundation of Kansas City (P.K.), and the Felix and Carmen Sabates Missouri Endowed Chair in Vision Research (P.K.). The content is solely the responsibility of the authors and does not necessarily represent the official views of the funding agencies.

\section{References}

American Cancer Society, (2011) Ovarian Cancer Reference literature, http:/ / www.cancer.org. Bignotti, E.; et al. (2007). Gene expression profile of ovarian serous papillary carcinomas: identification of metastasis-associated genes. Am. J. Obstet. Gynecol. 196:245.

Cooper, M. (2006). Current Biosensor Technologies in Drug Discovery. Drug Discovery World. 68-82.

Cunningham, A. (1998). Introduction to Bioanalytical Sensors. John Wiley and Sons, New York.

Cunningham, B.; Li, P.; Lin, B. \& Pepper, J. (2002). Colorimetric resonant reflection as a direct biochemical assay technique. Sens. Actuators B. 81:316-328.

Gaylord, T. K. and Moharam, M. G. (1985). Analysis and applications of optical diffraction by gratings. Proceedings of the IEEE. 73:894-937.

Homola, J. (2003). Present and future of surface plasmon resonance biosensors. Anal. Bioanal. Chem. 377:528-539.

Karlan, B.Y. \& Lagasse, L.D. (1994). Glucocorticoids stabilize HER-2/neu messenger RNA in human epithelial ovarian carcinoma cells. Gynecologic Oncology 53:70-77.

Kikuta, H.; Maegawa, N.; Mizutani, A.; Iwata, K.; \& Toyota, H. (2001). Refractive index sensor with a guided-mode resonant grating filter. Proceedings of the SPIE. Yokohama, Japan. 4416:219-222.

Liotta, L.; Lowenthal, M.; \& Mehta, A. (2005). Importance of communication between producers and consumers of publicly available experimental data. J. Natl. Cancer Inst. 97:310-314.

Magnusson, R. \& S. S. Wang. (1992). New principle for optical filters. Appl. Phys. Lett. 61:1022-1024.

Magnusson, R.; Wawro, D.; Zimmerman, S.; \& Ding, Y. (2011). Resonant Photonic Biosensors with Polarization-Based Multiparametric Discrimination in Each Channel, Sensors, 11, 1476-1488. 
Priambodo, P.S.; Maldonado, T.A. \& Magnusson, R. (2003). Fabrication and characterization of high-quality waveguide-mode resonant optical filters. Appl. Phys. Lett. 83, 32483250 .

Provencher, D.M.; et al; (1993). Comparison of antigen expression on fresh and cultured ascites cells and on solid tumors of patients with epithelial ovarian cancer. Gynecologic Oncology 50:1,78-83.

Raether, H. (1988). Surface plasmons on smooth and rough surfaces and on gratings. Springer Verlag, Berlin.

Rosenblatt, D.; Sharon, A; \& Friesem, A. (1997) Resonant Grating Waveguide Structures. IEEE J. Quantum Electron. 33, 2038.

Thermo Scientific Tech Tip \#58. (2007) Spike-and-recovery and linearity-of-dilution assessment. www.thermo.com

Wawro, D.; Tibuleac, S.; Magnusson, R.; \& Liu, H. (2000). Optical fiber endface biosensor based on resonances in dielectric waveguide gratings. Proceedings of the SPIE. San Jose, California. 3911:86-94.

Wawro, D.; Tibuleac, S.; \& Magnusson, R. (2006). Optical waveguide-mode resonant biosensors. Optical Imaging Sensors and Systems for Homeland Security Applications. 367-384.

Wawro, D.; Koulen, P.; Ding, Y.; Zimmerman, S. \& Magnusson, R. (2010). Guided-mode resonance sensor system for early detection of ovarian cancer. Optical Diagnostics and Sensing X: Toward Point-of-Care Diagnostics. Proceedings of the SPIE. San Francisco, California. 7572:75720D. 


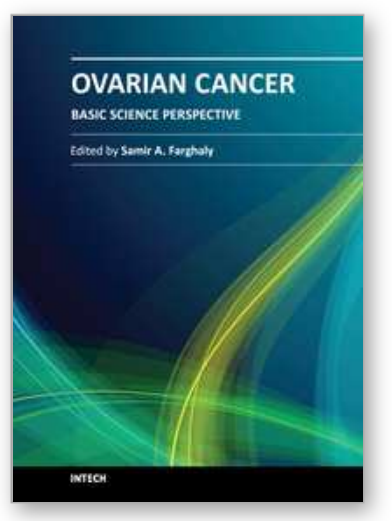

\author{
Ovarian Cancer - Basic Science Perspective \\ Edited by Dr. Samir Farghaly
}

ISBN 978-953-307-812-0

Hard cover, 406 pages

Publisher InTech

Published online 17, February, 2012

Published in print edition February, 2012

Worldwide, Ovarian carcinoma continues to be responsible for more deaths than all other gynecologic malignancies combined. International leaders in the field address the critical biologic and basic science issues relevant to the disease. The book details the molecular biological aspects of ovarian cancer. It provides molecular biology techniques of understanding this cancer. The techniques are designed to determine tumor genetics, expression, and protein function, and to elucidate the genetic mechanisms by which gene and immunotherapies may be perfected. It provides an analysis of current research into aspects of malignant transformation, growth control, and metastasis. A comprehensive spectrum of topics is covered providing up to date information on scientific discoveries and management considerations.

\title{
How to reference
}

In order to correctly reference this scholarly work, feel free to copy and paste the following:

Debra Wawro, Shelby Zimmerman, Robert Magnusson and Peter Koulen (2012). Photonic Sensor System for Screening Serum Biomarker Proteins in Ovarian Cancer, Ovarian Cancer - Basic Science Perspective, Dr. Samir Farghaly (Ed.), ISBN: 978-953-307-812-0, InTech, Available from: http://www.intechopen.com/books/ovarian-cancer-basic-science-perspective/portable-photonic-sensor-systemfor-screening-serum-biomarker-proteins-in-ovarian-cancer-

\section{INTECH}

open science | open minds

\author{
InTech Europe \\ University Campus STeP Ri \\ Slavka Krautzeka 83/A \\ 51000 Rijeka, Croatia \\ Phone: +385 (51) 770447 \\ Fax: +385 (51) 686166 \\ www.intechopen.com
}

\author{
InTech China \\ Unit 405, Office Block, Hotel Equatorial Shanghai \\ No.65, Yan An Road (West), Shanghai, 200040, China \\ 中国上海市延安西路 65 号上海国际贵都大饭店办公楼 405 单元 \\ Phone: +86-21-62489820 \\ Fax: +86-21-62489821
}


(C) 2012 The Author(s). Licensee IntechOpen. This is an open access article distributed under the terms of the Creative Commons Attribution 3.0 License, which permits unrestricted use, distribution, and reproduction in any medium, provided the original work is properly cited. 